Harold J. Burstein, Eric P. Winer, DanaFarber Cancer Institute, Boston, MA; Sarah Temin, American Society of Clinical Oncology, Alexandria, VA; Holly Anderson, Breast Cancer Coalition of Rochester; Alexander J. Solky, Interlakes Onc and Hem PC, Rochester; Clifford A. Hudis, Memoria Sloan Kettering Cancer Center, New York, NY; Thomas A. Buchholz, Sharon H. Giordano, The University of Texas MD Anderson Cancer Center, Houston; Diana Rowden, Dallas, TX; Nancy E. Davidson, University of Pittsburgh Cancer Institute and UPMC Cancer Center, Pittsburgh, PA Karen E. Gelmon, British Columbia Cancer Agency, Vancouver, British Columbia, Canada; Vered Stearns, Johns Hopkins School of Medicine, Baltimore, MD; and Jennifer J. Griggs, University of Michigan, Ann Arbor, Ml.

Published online ahead of print at www.jco.org on May 27, 2014

Editor's note: This American Society of Clinical Oncology (ASCO) Clinical Practice Guideline provides recommendations,

with review and analyses of the relevant literature for each recommendation. Additional information, which may include data supplements, slide sets, patient versions, clinical tools and resources, is available at www.asco.org/guidelines/ endocrinebreast.

Authors' disclosures of potential conflicts of interest and author contributions are found at the end of this article.

Reprint Requests: 2318 Mill Rd, Suite 800 Alexandria, VA 22314; guidelines@asco.org.

Authors' disclosures of potential conflicts of interest and author contributions are found at the end of this article.

Clinical Practice Guideline Committee Approved: January 31, 2014

Corresponding author: Harold J. Burstein, MD, PhD, c/o American Society of Clinical Oncology, 2318 Mill Rd, Suite 800, Alexandria, VA 22314;

e-mail: guidelines@asco.org

(C) 2014 by American Society of Clinical Oncology

0732-183X/14/3221w-2255w/\$20.00

DOI: 10.1200/JCO.2013.54.2258

\title{
Adjuvant Endocrine Therapy for Women With Hormone Receptor-Positive Breast Cancer: American Society of Clinical Oncology Clinical Practice Guideline Focused Update
}

Harold J. Burstein, Sarah Temin, Holly Anderson, Thomas A. Buchholz, Nancy E. Davidson, Karen E. Gelmon, Sharon H. Giordano, Clifford A. Hudis, Diana Rowden, Alexander J. Solky, Vered Stearns, Eric P. Winer, and Jennifer J. Griggs

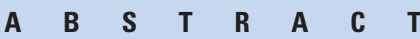

\section{Purpose}

To update the ASCO clinical practice guideline on adjuvant endocrine therapy on the basis of emerging data on the optimal duration of treatment, particularly adjuvant tamoxifen.

\section{Methods}

ASCO convened the Update Committee and conducted a systematic review of randomized clinical trials from January 2009 to June 2013 and analyzed three historical trials. Guideline recommendations were based on the Update Committee's review of the evidence. Outcomes of interest included survival, disease recurrence, and adverse events.

\section{Results}

This guideline update reflects emerging data on duration of tamoxifen treatment. There have been five studies of tamoxifen treatment beyond 5 years of therapy. The two largest studies with longest reported follow-up show a breast cancer survival advantage with 10-year durations of tamoxifen use. In addition to modest gains in survival, extended therapy with tamoxifen for 10 years compared with 5 years was associated with lower risks of breast cancer recurrence and contralateral breast cancer.

\section{Recommendations}

Previous ASCO guidelines recommended treatment of women who have hormone receptorpositive breast cancer and are premenopausal with 5 years of tamoxifen, and those who are postmenopausal a minimum of 5 years of adjuvant therapy with an aromatase inhibitor or tamoxifen followed by an aromatase inhibitor (in sequence). If women are pre- or perimenopausal and have received 5 years of adjuvant tamoxifen, they should be offered 10 years total duration of tamoxifen. If women are postmenopausal and have received 5 years of adjuvant tamoxifen, they should be offered the choice of continuing tamoxifen or switching to an aromatase inhibitor for 10 years total adjuvant endocrine therapy.

\section{J Clin Oncol 32:2255-2269. (C) 2014 by American Society of Clinical Oncology}

\section{INTRODUCTION}

Hormone receptor-positive breast cancers represent the vast majority of breast cancers around the world. Approximately $60 \%$ to $75 \%$ of women with breast cancer have estrogen receptor-positive $(\mathrm{ER}+)$ breast cancer, and $65 \%$ of these cancers are also progesterone receptor positive $(\mathrm{PgR}+) .{ }^{1-4} \mathrm{Ad}-$ juvant endocrine therapy is highly effective and appropriate for nearly all women with ER- and/or PgR-positive tumors, making such treatment the most widely prescribed therapy for patients with cancer in both the developed and developing world. For decades, tamoxifen taken for 5 years was the standard adjuvant endocrine treatment. More recently, patients who are postmenopausal also have been offered the option of taking an aromatase inhibitor (AI) as an alternative to tamoxifen or in sequence after tamoxifen. ${ }^{5}$

Adjuvant Endocrine Therapy for Women With Hormone Receptor-Positive Breast Cancer: American Society of Clinical Oncology Clinical Practice Guideline Focused Update is an update of previous technology assessment/guideline updates from 2002, 2003, 2004, and 2010 (www.asco.org/ guidelines/endocrinebreast). ${ }^{5-8}$ The current update is in response to emerging data from multinational randomized studies that addressed the 


\section{Adjuvant Endocrine Therapy for Women with Hormone Receptor-Positive Breast Cancer: American Society of Clinical Oncology Clinical Practice Guideline Focused Update}

\section{Guideline Question}

- What is the recommended duration of adjuvant endocrine monotherapy?

\section{Target Audience}

- Medical, surgical, and radiation oncologists; oncology nurses and physician assistants; obstetrician/gynecologists; general practitioners; and women with stages I-III hormone receptor-positive breast cancer

\section{Methods}

- Update Committee was convened to develop clinical practice guideline recommendations based on a systematic review of the medical literature.

\section{Recommendations}

- I. Women diagnosed with hormone receptor-positive breast cancer who are pre- or perimenopausal should be offered adjuvant endocrine therapy with:

- IA. Tamoxifen for an initial duration of 5 years.

- IB. After 5 years, women should receive additional therapy based on menopausal status.

- IB1. If women are pre- or perimenopausal, or if menopausal status is unknown or cannot be determined, they should be offered continued tamoxifen for a total duration of 10 years (Type: Evidence-Based, Evidence Quality: High, Strength of Recommendation: Strong).

- IB2. If women have become definitively postmenopausal, they should be offered continued tamoxifen for a total duration of 10 years or switching to up to 5 years of an aromatase inhibitor (AI), for a total duration of up to 10 years of adjuvant endocrine ther apy. (Type: Evidence-Based, Evidence Quality for tamoxifen: High, Evidence Quality for AI: High; Strength of Recommendation: Strong)

- II. Women diagnosed with hormone receptor-positive breast cancer who are postmenopausal should be offered adjuvant endocrine therapy with one of the following options:

- IIA. Tamoxifen for a duration of 10 years (Type: Evidence-Based, Evidence Quality: High, Strength of Recommendation: Strong); or

- IIB. An AI for a duration of 5 years. There are insufficient data currently to recommend an AI for a duration of greater than 5 years. (Type: Evidence-Based, Evidence Quality: High, Strength of Recommendation: Strong); or

- IIC. Tamoxifen for an initial duration of 5 years, then switching to an AI for up to 5 years, for a total duration of up to 10 years of adjuvant endocrine therapy. (Type: Evidence-Based, Evidence Quality: High, Strength of recommendation: Strong); or

- IID. Tamoxifen for a duration of 2 to 3 years and switching to an AI for up to 5 years, for a total duration of up to 7 to 8 years of adjuvant endocrine therapy. (Type: Evidence-Based, Evidence Quality: High, Strength of Recommendation: Strong)

- III. Women who are postmenopausal and are intolerant of either tamoxifen or an AI should be offered the alternative type of adjuvant endocrine therapy.

- IIIA. If women have received an AI but discontinued treatment at less than 5 years, they may be offered tamoxifen for a total of 5 years. (Type: Informal consensus, Evidence Quality: Low, Strength of Recommendation: Weak)

- IIIB. If women have received tamoxifen for 2 to 3 years, they should be offered switching to an AI for up to 5 years, for a total duration of up to 7 to 8 years of adjuvant endocrine therapy. (Type: Evidence-Based, Evidence Quality: High, Strength of Recommendation: Strong)

- IV. Women who have received 5 years of tamoxifen as adjuvant endocrine therapy should be offered additional adjuvant endocrine treatment.

- IVA. If women are postmenopausal, they should be offered continued tamoxifen for a total duration of 10 years or switching to up to 5 years AI, for a total duration of up to 10 years of adjuvant endocrine therapy. (Type: Evidence-Based, Evidence Quality: High, Strength of Recommendation: Strong)

- IVB. If women are pre- or perimenopausal, or menopausal status cannot be ascertained, they should be offered 5 additional years of tamoxifen, for a total duration of 10 years of adjuvant endocrine therapy. (Type: Evidence-Based, Evidence Quality: High, Strength of Recommendation: Strong)

(continued on following page) 
THE BOTTOM LINE (CONTINUED)

\section{Tradeoffs of Benefits and Risks}

- Benefits: increasing overall survival and distant disease-free survival (DFS), reducing breast cancer-specific mortality, decreased risk of recurrence, decreased risk of contralateral breast cancer

- Harms: endometrial cancer (if continuing tamoxifen), hot flashes and other menopausal symptoms (with either tamoxifen or AIs), deep vein thrombosis or pulmonary embolism (tamoxifen), ischemic heart disease (AI), osteopenia/osteoporosis (AI), uterine cancer (tamoxifen)

\section{Qualifying Statements}

- There are no specific patient populations or subgroups, except for menopausal status, that derive differing degrees of benefit from an $\mathrm{AI}$ versus tamoxifen or for the durations discussed above. Clinicians and patients should discuss a patient's individual riskbenefit profiles (see Commentary section for further discussion).

\section{Key Changes}

- Tamoxifen is now recommended for a duration of up to 10 years rather than 5 years.

\section{ADDITIONAL RESOURCES}

- More information, including data supplements with additional evidence tables, a Methodology Supplement with information about evidence quality and strength of recommendations, slide sets, and clinical tools and resources, is available at www.asco.org/ guidelines/endocrinebreast. Patient information is at www.cancer.net.

duration of endocrine therapy. This includes two randomized trials-one published and the other presented at the 2013 ASCO Annual Meeting - on extended durations of tamoxifen treatment. ${ }^{9,10}$ The 2010 guideline update referred to a historical standard of 5-year duration of tamoxifen monotherapy, because earlier studies of extended tamoxifen therapy had not shown clinical benefit for treatment beyond 5 years. The current guideline update reviews and analyzes the new data regarding that aspect of the guideline.

This Focused Update addresses only duration of tamoxifen monotherapy. The Update does not address the other clinical questions posed in the 2010 guideline. The Bottom Line Box provides a summary of the recommendations. A comparison of 2010 and current recommendations is available in the Data Supplement.

\section{GUIDELINE QUESTIONS}

This clinical practice guideline addresses three overarching clinical questions: (1) Which adjuvant endocrine treatments should be offered to women with hormone receptor-positive breast cancer? (2) What is the appropriate duration of adjuvant endocrine therapy? (3) What is the appropriate sequence of adjuvant endocrine therapy?

\section{METHODS}

The recommendations for this update were developed by a multidisciplinary group of experts using evidence from phase III randomized clinical trials (RCTs) and clinical experience as a guide. Clinical Practice Guideline Updates are approved by the ASCO Clinical Practice Guideline Committee. A systematic review in Medline was conducted from January 2009 to March 2013 and for meeting abstracts through June 2013. Because this update addressed a question not specifically identified in previous updates, the search date parameters were broadened to find historical trials.

Articles were selected for inclusion in the systematic review of the evidence if they were

- Published journal articles from the medical literature.

- Phase III RCTs.

- Meeting abstracts, if presentations or posters were available.

- Written language: English only.

- Systematic reviews with or without meta-analysis.

- Study population: female.

Articles were excluded from the systematic review if they were (1) other reviews (consensus, narrative, expert panel, guidelines); (2) editorials, commentaries, letters, news articles, case reports; or (3) published in a non-English language. The guideline recommendations were crafted, in part, using the Guidelines Into Decision Support (GLIDES) methodology and accompanying BRIDGE-Wiz software. ${ }^{11}$ Ratings for the type of recommendation, strength of the evidence, and potential bias are provided with each recommendation (see Methodology Supplement for rating definitions).

Detailed information about the methods used to develop this guideline is available in the Methodology Supplement at www.asco.org/guidelines/endocrinebreast, including an overview (eg, Update Committee composition, development process), literature search and data extraction, the recommendation development (including GLIDES and BRIDGEWiz), and quality assessment processes.

\section{GUIDELINE DISCLAIMER}

The clinical practice guidelines and other guidance published herein are provided by the American Society of Clinical Oncology, Inc. ("ASCO") to assist providers in clinical decision making. The information therein should not be relied on as being complete or accurate, 
nor should it be considered as inclusive of all proper treatments or methods of care or as a statement of the standard of care. With the rapid development of scientific knowledge, new evidence may emerge between the time information is developed and when it is published or read. The information is not continually updated and may not reflect the most recent evidence. The information addresses only the topics specifically identified therein and is not applicable to other interventions, diseases, or stages of diseases. This information does not mandate any particular course of medical care. Further, the information is not intended to substitute for the independent professional judgment of the treating provider, as the information does not account for individual variation among patients. Recommendations reflect high, moderate or low confidence that the recommendation reflects the net effect of a given course of action. The use of words like "must," "must not," "should," and "should not" indicate that a course of action is recommended or not recommended for either most or many patients, but there is latitude for the treating physician to select other courses of action in individual cases. In all cases, the selected course of action should be considered by the treating provider in the context of treating the individual patient. Use of the information is voluntary. ASCO provides this information on an "as is" basis, and makes no warranty, express or implied, regarding the information. ASCO specifically disclaims any warranties of merchantability or fitness for a particular use or purpose. ASCO assumes no responsibility for any injury or damage to persons or property arising out of or related to any use of this information or for any errors or omissions.

\section{GUIDELINE AND CONFLICT OF INTEREST}

The Update Committee was assembled in accordance with ASCO's Conflict of Interest Management Procedures for Clinical Practice Guidelines ("Procedures," summarized at http://www.asco.org/ rwc). Members of the Update Committee completed ASCO's disclosure form, which requires disclosure of financial and other interests that are relevant to the subject matter of the guideline, including relationships with commercial entities that are reasonably likely to experience direct regulatory or commercial impact as the result of promulgation of the guideline. Categories for disclosure include employment relationships, consulting arrangements, stock ownership, honoraria, research funding, and expert testimony. In accordance with the Procedures, the majority of the members of the Update Committee did not disclose any such relationships.

\section{RESULTS}

Note: This guideline update reviews only evidence on durations of tamoxifen of greater than 5 years. Evidence regarding duration of adjuvant endocrine therapy 5 years or less, evidence regarding sequencing of therapy, and evidence on AIs is reviewed in the 2010 guideline update, to which the reader is referred. ${ }^{5}$

Five RCTs met the eligibility criteria of the updated systematic review and compose the evidentiary basis for the guideline recommendations on the duration of tamoxifen therapy (see Data Supplement 1). ${ }^{9,10,12-15}$ Each of these studies compared 5 years of tamoxifen against longer durations: either indefinitely or for a total of 10 years.
Data from the three smaller trials have been in the literature for more than a decade; data from two studies represent results available only recently. The identified trials' recruitment periods spanned from 1982 to 2005, a 23-year period, and the publication dates spanned from 1996 to 2013, a 17-year period. The trials compared similar interventions. The primary outcome for all trials was therapeutic efficacy, defined variously as overall survival (OS), DFS, recurrence-free survival (RFS), time to recurrence (TRR), event rate ratio (ERR), and average annual rate of recurrence. Adverse events were a secondary outcome of interest.

\section{STUDY QUALITY}

Study quality was formally assessed for the five studies identified (Table 1). Aspects of trial design related to the individual study quality were assessed by one reviewer using factors such as blinding, allocation concealment, placebo control, intention to treat, and funding sources. The risk of bias was assessed as low or intermediate, or it was not possible to assess this for the identified evidence. Refer to the Methodology Supplement for definitions of ratings for overall potential risk of bias.

\section{RECOMMENDATIONS}

\section{Clinical Question I}

Which adjuvant endocrine treatments should be offered to women with hormone receptor-positive breast cancer who are pre- or perimenopausal? What is the appropriate duration?

Recommendation 1. Women diagnosed with hormone receptorpositive breast cancer who are pre- or perimenopausal should be offered adjuvant endocrine therapy with:

Recommendation IA. Tamoxifen for an initial duration of 5 years (supported by 2010 evidence).

Recommendation IB. After 5 years, women should receive additional therapy based on menopausal status.

- Recommendation IB1. If women are pre- or perimenopausal, or if menopausal status is unknown or cannot be determined, they should be offered continued tamoxifen for a total duration of 10 years. (Type: Evidence-Based, Evidence Quality: High, Strength of Recommendation: Strong; supported by 2013 evidence, see Literature Review section).

- Recommendation IB2. If women have become definitively postmenopausal, they should be offered the choice of continuing tamoxifen for a total duration of 10 years or switching to up to 5 years of an $\mathrm{AI}$, for a total duration of up to 10 years of adjuvant endocrine therapy. (Type: Evidence-Based, Evidence Quality for tamoxifen: High, Evidence Quality for AI: High; Strength of Recommendation: Strong; supported by 2010 and 2013 evidence).

\section{Clinical Question II}

Which adjuvant endocrine treatments should be offered to women with hormone receptor-positive breast cancer who are postmenopausal? What is the appropriate duration?

Recommendation II. Women diagnosed with hormone receptorpositive breast cancer who are postmenopausal should be offered adjuvant endocrine therapy with one of the following initial options: 


\begin{tabular}{|c|c|c|c|c|c|c|c|c|c|c|}
\hline $\begin{array}{l}\text { Study, } \\
\text { Author, } \\
\text { Year }\end{array}$ & $\begin{array}{c}\text { Adequate } \\
\text { Randomization }\end{array}$ & $\begin{array}{l}\text { Concealed } \\
\text { Allocation }\end{array}$ & $\begin{array}{l}\text { Sufficient } \\
\text { Sample } \\
\text { Size }\end{array}$ & Similar Groups & Blinded & $\begin{array}{l}\text { Validated } \\
\text { Measures }\end{array}$ & $\begin{array}{l}\text { Adequate } \\
\text { Follow-Up }\end{array}$ & $\begin{array}{l}\text { Intention-to- } \\
\text { Treat } \\
\text { Analysis }\end{array}$ & $\begin{array}{l}\text { Insignificant } \\
\mathrm{COI}\end{array}$ & $\begin{array}{l}\text { Overall } \\
\text { Potential } \\
\text { Risk of } \\
\text { Bias }^{a}\end{array}$ \\
\hline ECOG, Tormey, 1996 & $+^{b}$ & + & $?$ & + & - & $?$ & + & $?$ & + & Intermediate \\
\hline $\begin{array}{l}\text { NSABP B-14, Fisher, } \\
2001\end{array}$ & Partial $^{\mathrm{C}}$ & $?$ & $?$ & $+^{d}$ & + & + & $+^{e}$ & $-^{f}$ & + & Intermediate \\
\hline $\begin{array}{c}\text { Scottish Cancer Trials } \\
\text { Breast Group, } \\
\text { Stewart, } 2001\end{array}$ & + & $?$ & $?^{9}$ & $\begin{array}{l}+ \text { (at second } \\
\text { randomization) }\end{array}$ & - & $+^{h}$ & + & + & + & Intermediate \\
\hline ATLAS, Davies, 2012 & + & + & + & + & - & + & + & + & + & Low \\
\hline aTTom, Gray, 2013 & & NR & NR & NR & NR & NR & NR & NR & NR & $\begin{array}{l}\text { Insufficient } \\
\text { data } \\
\text { available } \\
\text { for } \\
\text { assessment }\end{array}$ \\
\hline \multicolumn{11}{|c|}{ 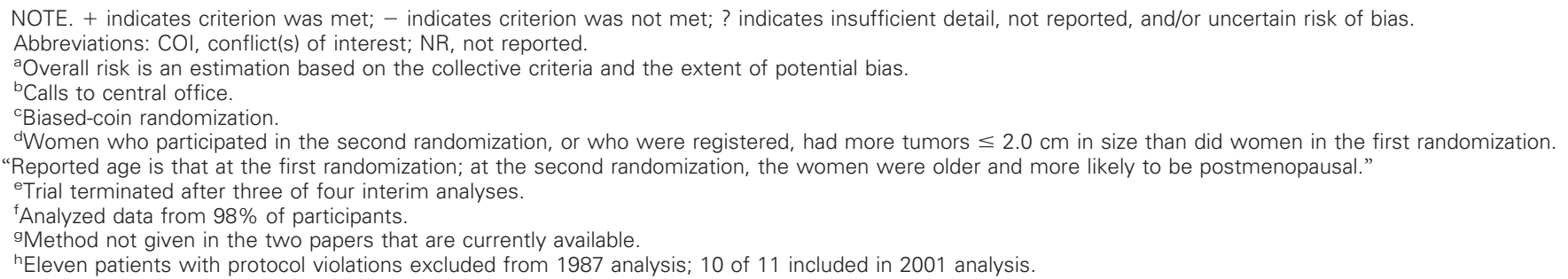 } \\
\hline
\end{tabular}

Recommendation IIA. Tamoxifen for a duration of 10 years. (Type: Evidence-Based, Evidence Quality: High, Strength of Recommendation: Strong; supported by 2013 evidence); or

Recommendation IIB. An AI for a duration of 5 years. There are insufficient data currently to recommend an AI for a duration of greater than 5 years. (Type: Evidence-Based, Evidence Quality: High, Strength of Recommendation: Strong; supported by 2010 evidence); or

Recommendation IIC. Tamoxifen for an initial duration of 5 years, then switching to an $\mathrm{AI}$ for up to 5 years, for a total duration of up to 10 years of adjuvant endocrine therapy. (Type: Evidence-Based, Evidence Quality: High, Strength of Recommendation: Strong; supported by 2010 evidence); or

Recommendation IID. Tamoxifen for a duration of 2 to 3 years and switching to an AI for up to 5 years, for a total duration of up to 7 to 8 years of adjuvant endocrine therapy. (Type: Evidence-Based, Evidence Quality: High, Strength of Recommendation: Strong; supported by 2010 evidence).

\section{Clinical Question III}

What is the appropriate sequence of adjuvant endocrine therapy?

Recommendation III. Women who are postmenopausal and are intolerant of either tamoxifen or an AI should be offered the alternative type of adjuvant endocrine therapy.

Recommendation IIIA. If women have received an AI but discontinued treatment at less than 5 years, they may be offered tamoxifen for a total of 5 years. (Type: Informal consensus, Evidence Quality: Low, Strength of Recommendation: Weak; supported by 2010 evidence).

Recommendation IIIB. If women have received tamoxifen for 2 to 3 years, they should be offered the option of switching to an AI for up to 5 years, for a total duration of up to 7 to 8 years of adjuvant endocrine therapy. (Type: Evidence-Based, Evidence Quality: High,
Strength of Recommendation: Strong; supported by 2010 evidence; see also Recommendations IB2, IIC, and IID and III).

Recommendation IV. Women who have received 5 years of tamoxifen as adjuvant endocrine therapy should be offered additional adjuvant endocrine treatment.

Recommendation IVA. If women are postmenopausal, they should be offered continued tamoxifen for a total duration of 10 years or the option of switching to up to 5 years of an AI, for a total duration of up to 10 years of adjuvant endocrine therapy. (Type: EvidenceBased, Evidence Quality: High, Strength of Recommendation: Strong; supported by 2010 and 2013 evidence)

Recommendation IVB. If women are pre- or perimenopausal, or menopausal status cannot be ascertained, they should be offered 5 additional years of tamoxifen, for a total of 10 years of adjuvant endocrine therapy. (Type: Evidence-Based, Evidence Quality: High, Strength of Recommendation: Strong; supported by 2013 evidence).

\section{LITERATURE REVIEW AND ANALYSIS}

This section summarizes the efficacy results for durations of tamoxifen greater than 5 years, based on five prospective, randomized trials that were considered in the systematic review for this clinical question (regarding tamoxifen in Recommendations IB1, IB2, IIA, IVA, and IVB $^{9,10,12,14,15}$; Tables 2 and 3 include results from these trials).

\section{OS and Mortality}

All five studies reported on OS and mortality, although not all populations in all studies had tumors with known ER status. Survival outcomes were mixed. The two more recent, larger studies suggested a survival benefit for longer durations of tamoxifen. In the ATLAS (Adjuvant Tamoxifen Longer Against Shorter) trial, the breast 


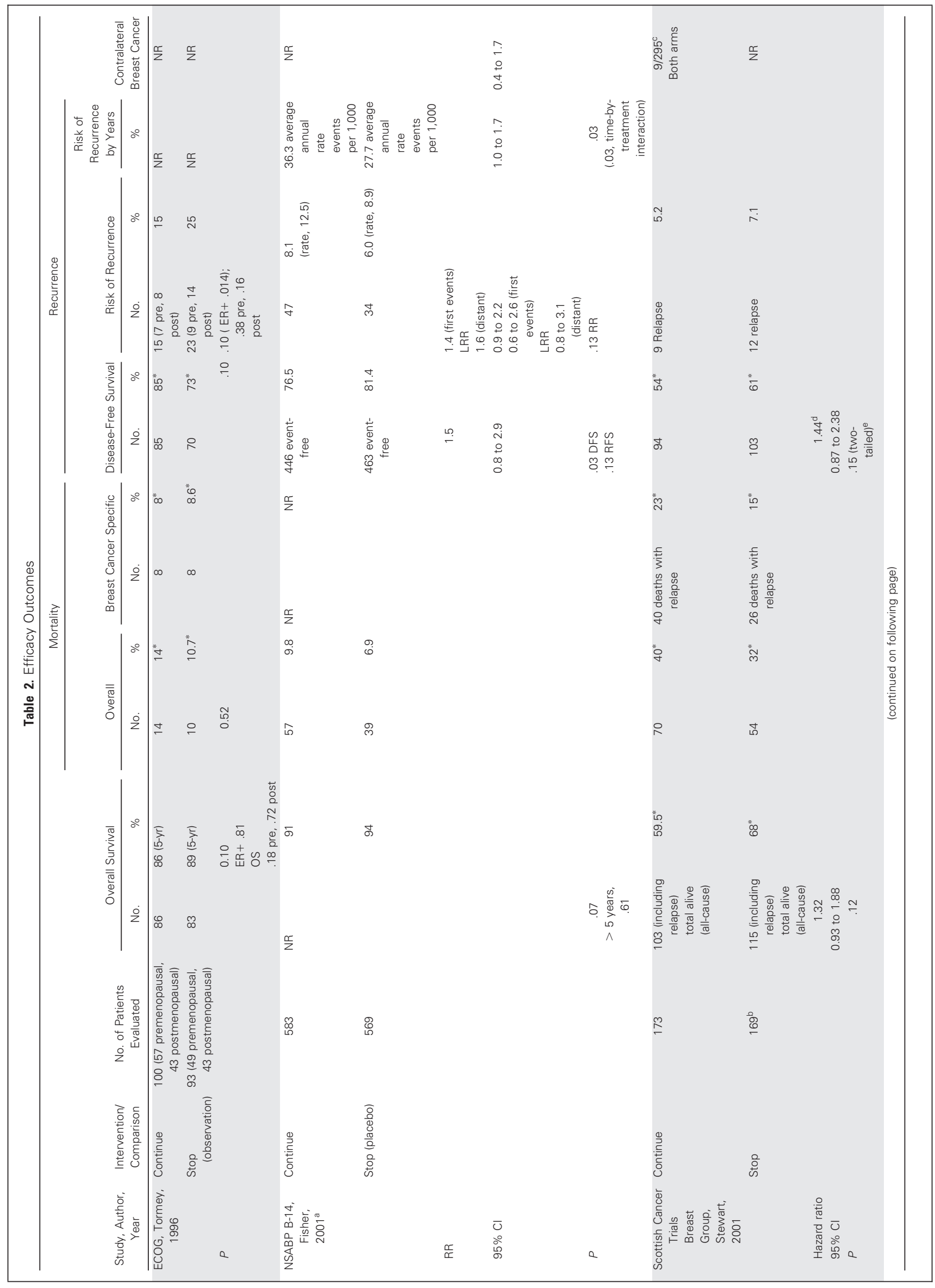




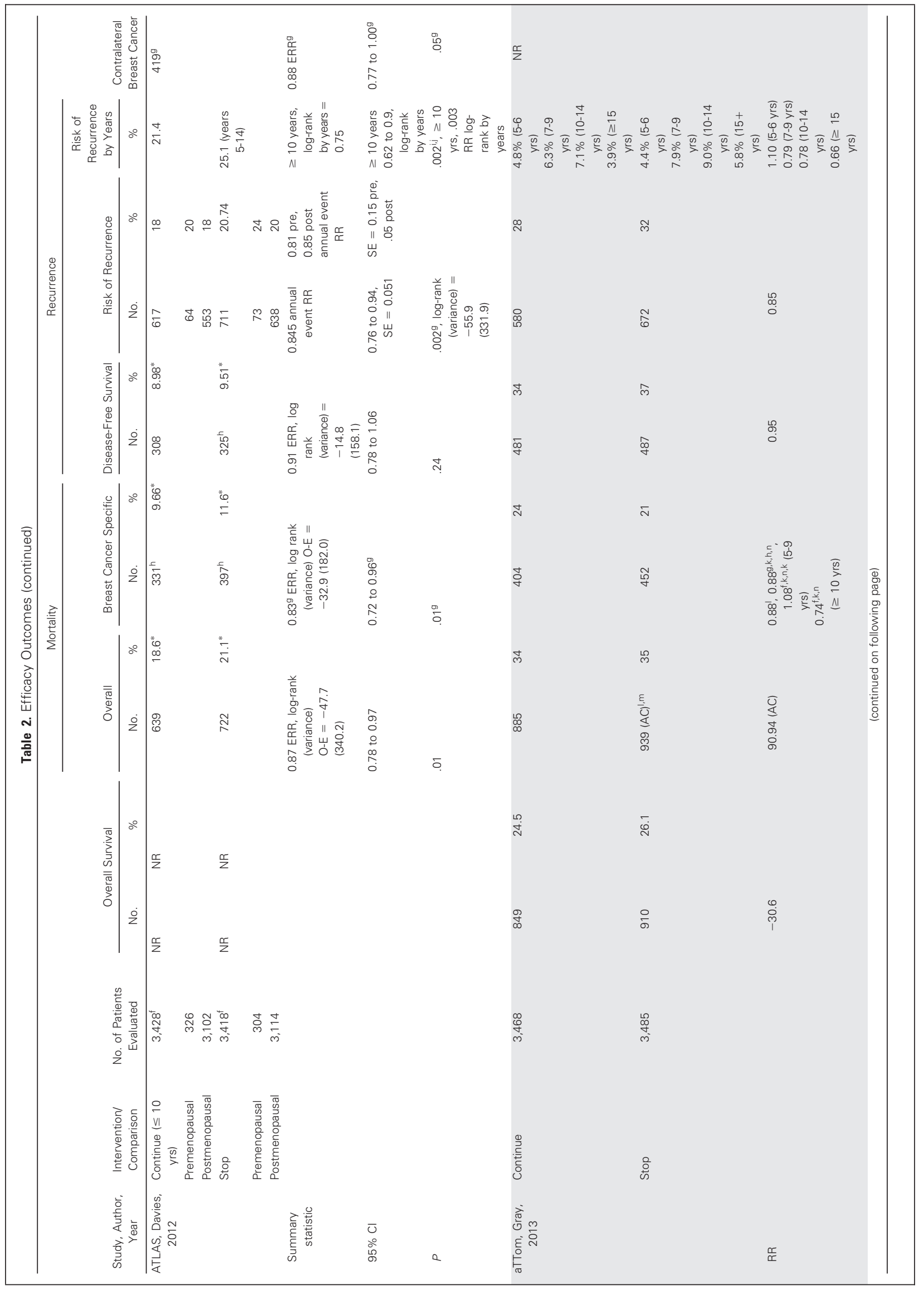




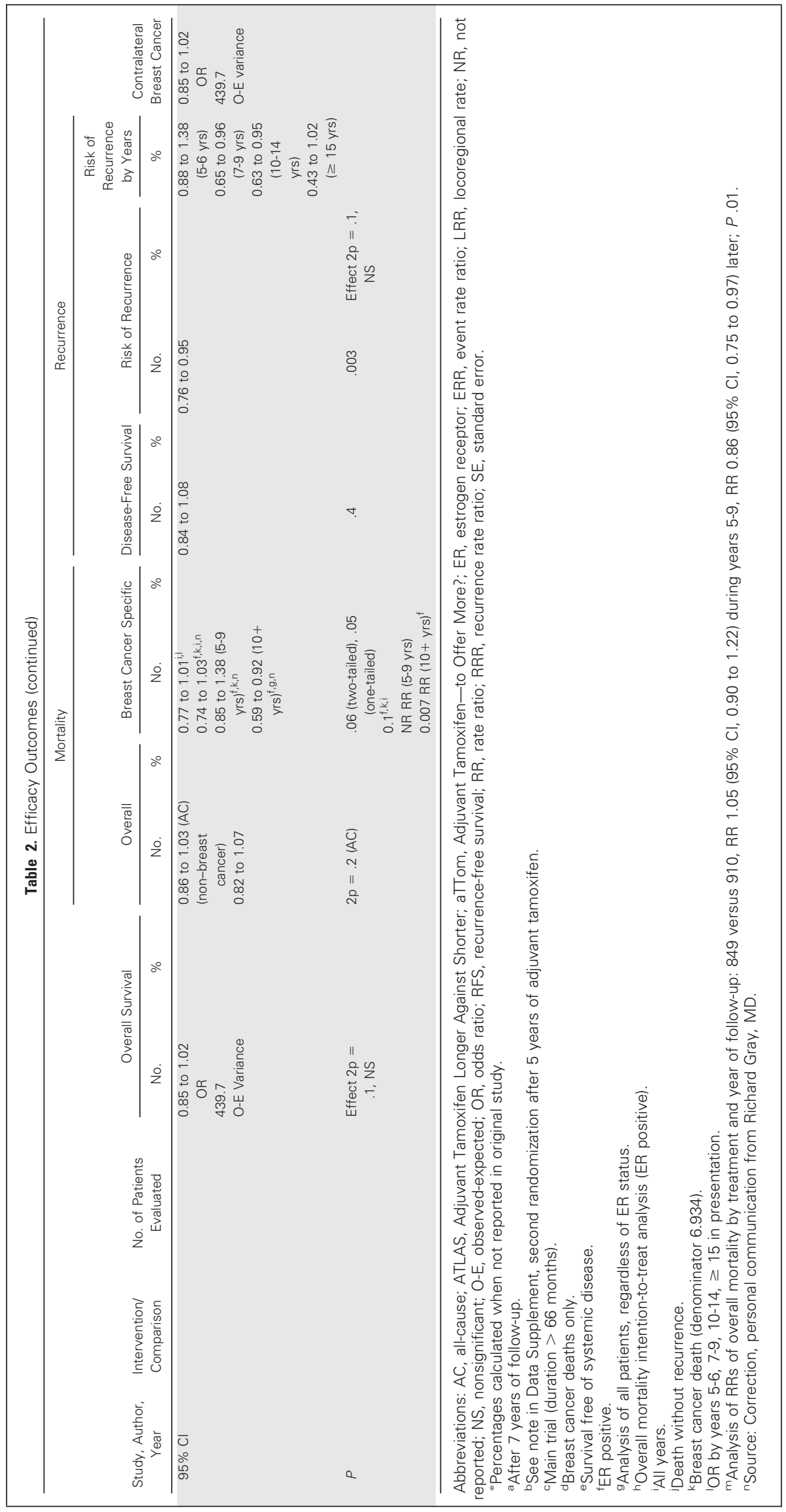




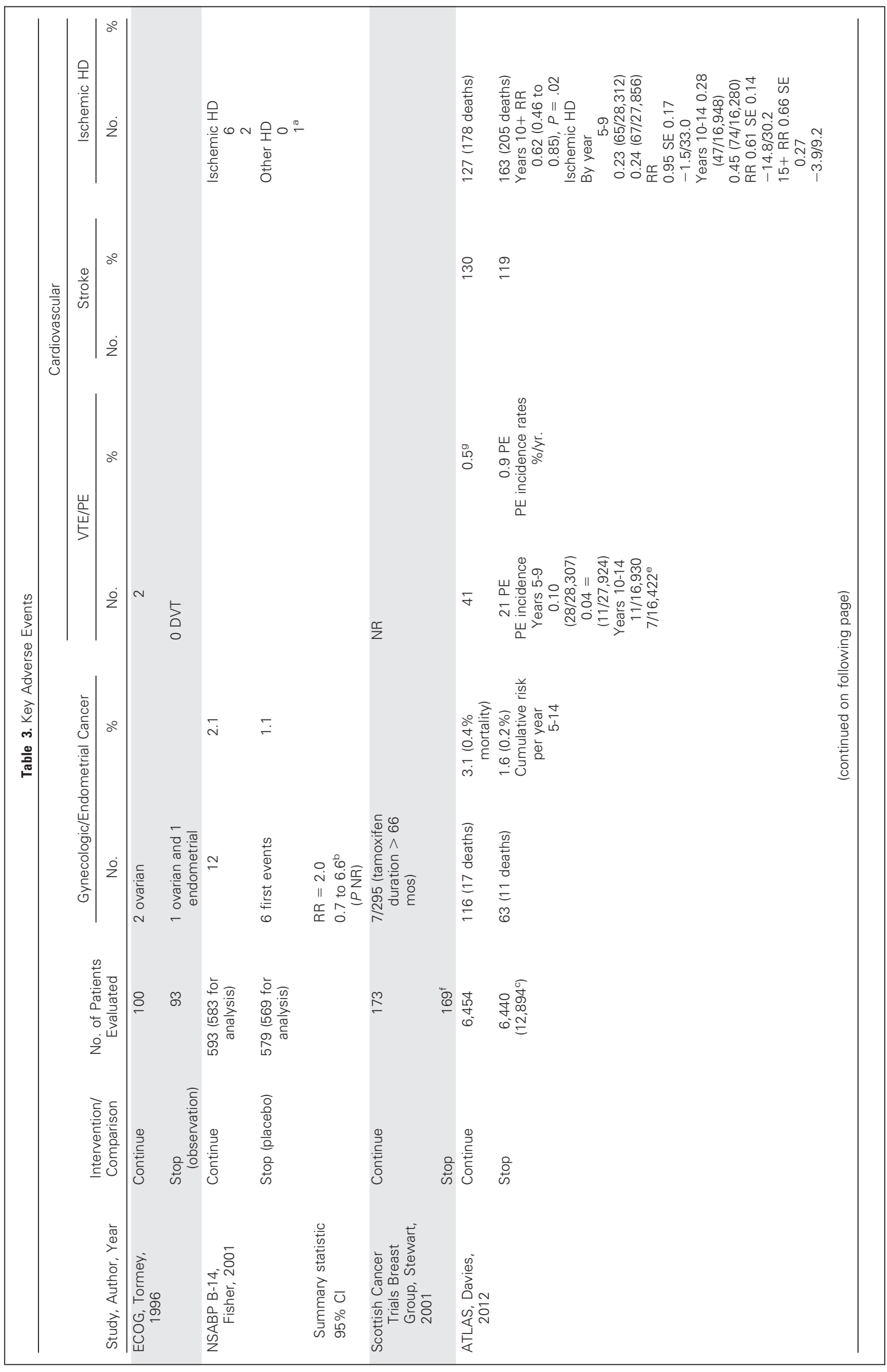




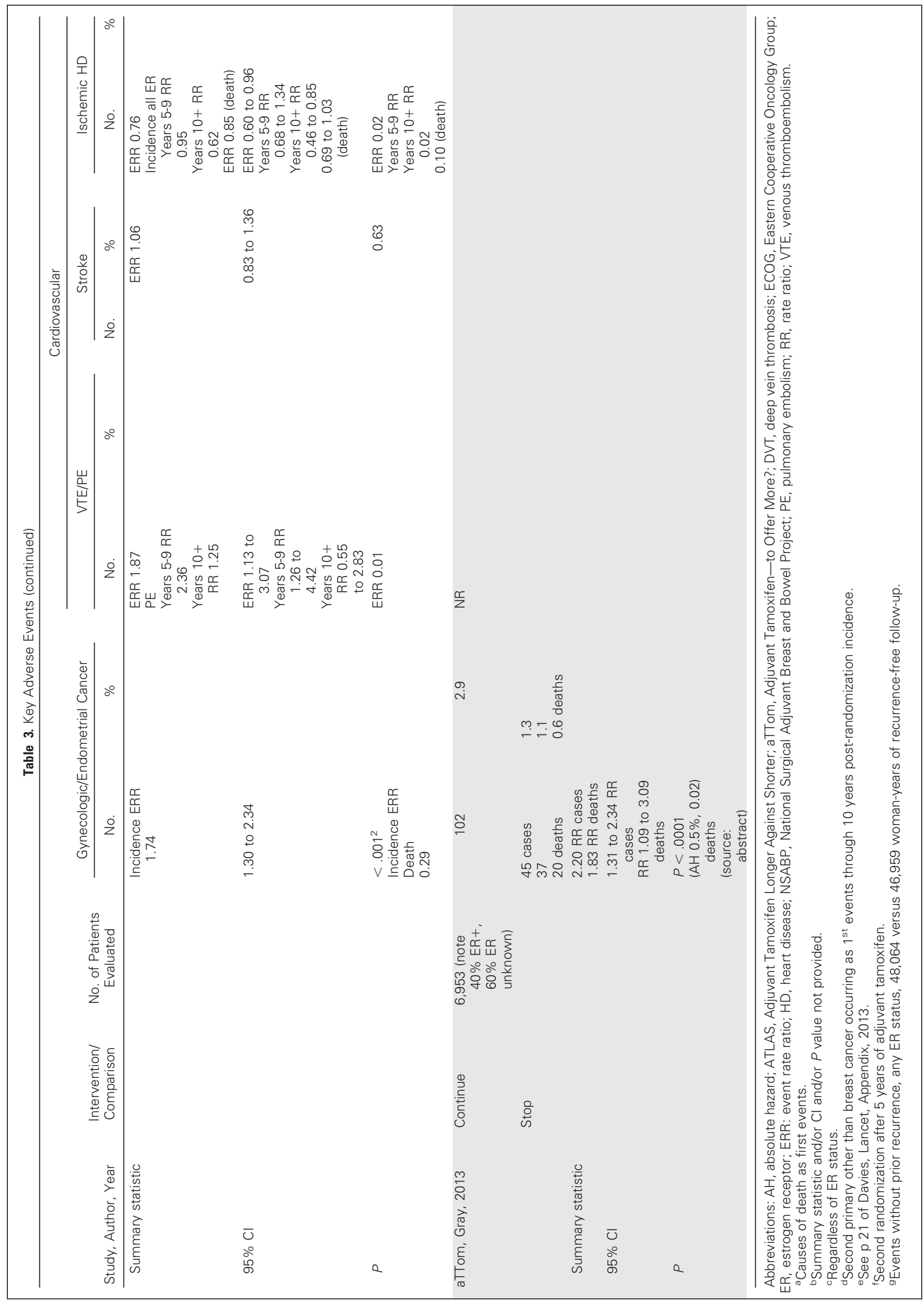


cancer-specific mortality was $2.8 \%$ lower in those who took tamoxifen for 10 years $(P=.01)$, compared with those who took tamoxifen for 5 years $(E R R=0.83 ; 95 \% C I, 0.72$ to 0.96$)$. Similarly, breast cancer-specific survival was improved with extended tamoxifen treatment in the aTTom (Adjuvant Tamoxifen; to Offer More?) trial $^{10}$ and in the historical Eastern Cooperative Oncology Group trial. ${ }^{15}$ Overall mortality was improved in three of the five trials. ${ }^{9,10,15}$ However, mortality was higher in the extended tamoxifen arm in the other two historical studies. ${ }^{12,13}$ In National Surgical Adjuvant Breast and Bowel Project (NSABP) trial B14, the results were measured at greater than 5 years. $^{12}$ (Table 2$)$.

\section{DFS and RFS}

All five studies reported on recurrences, using measures including DFS, recurrence- or relapse-free survival, and time to recurrence. In the ATLAS and aTTom trials, extended tamoxifen therapy lowered the risk of breast cancer recurrence. This was also true in the ERpositive subset of the Eastern Cooperative Oncology Group trial. However, in both the smaller Scottish and NSABP B-14 trials, extended therapy with tamoxifen was associated with greater risk of cancer recurrence and/or shortened DFS.

\section{Risk of Recurrence by Years}

Three studies analyzed recurrence rates as a function of the length of follow-up from time of study enrollment (of the participants who had taken tamoxifen beyond 5 years). In the ATLAS trial, the recurrence rate risk ratio (RR) was significantly lower with longer durations, at $21.4 \%$ for $\geq 10$ years ( $R R=0.75 ; 95 \% \mathrm{CI}, 0.62$ to 0.0 .9 ) versus $25.1 \%$ for $5-9$ years $(\mathrm{RR}=0.90 ; 95 \% \mathrm{CI}, 0.79$ to $1.02 ; P=.002) .{ }^{9}$ For the aTTom trial, in a preplanned analysis of recurrence by year of followup, the benefit of additional tamoxifen was statistically significant in years 7 to 9 and years 10 to 14, favoring continuation (beyond 5 years), whereas for shorter or longer time periods, the difference was not statistically significant. ${ }^{10}$ In another study, which did not favor tamoxifen, the time-by-treatment interaction was statistically significant $(P=.03)$; there was statistically significant greater recurrence in years zero to 5 years and not in $\geq 6$ years. ${ }^{12}$

\section{Contralateral Breast Cancer}

Three of five studies reported the incidence of contralateral breast cancer. ${ }^{9,12,14}$ One study showed that the risk was $12 \%$ lower with tamoxifen, $(\mathrm{RR}=0.88 ; 95 \% \mathrm{CI}, 0.77 \text { to } 1.00 ; P=.05)^{9}$ in analysis of all patients, regardless of ER status, whereas the NSABP B-14 and Scottish studies showed no statistically significant differences in rates of contralateral breast cancer between patient populations.

\section{Adverse Events}

All five studies reported on adverse events. There are well-described adverse effects with extended duration therapy with tamoxifen, all of which had been identified in previous reports. ${ }^{5-8}$ Endometrial cancer was significantly more common among women who received extended therapy with tamoxifen compared with those who did not: $3.1 \%$ versus $1.6 \%{ }^{9}$ (cumulative risk) and $2.9 \%$ versus $1.3 \%$ (incidence),${ }^{10}$ in ATLAS and aTTom, respectively. The numerical instances of death as a result of endometrial cancer were also higher with extended tamoxifen treatment, though such events were rare. There was also a higher risk for pulmonary embolism with tamoxifen. There was a lower risk of ischemic heart disease in women who received extended tamoxifen therapy in ATLAS. ${ }^{9}$ NSABP B-14 reported an increased risk for ischemic heart disease, but there were few events. ${ }^{12}$ Rates of stroke were similar in the only study that reported on it. ${ }^{9}$ None of the five studies reported other adverse events considered germane to the Update Committee's deliberations, including menopausal symptoms (Table 3).

\section{Quality of Life}

None of the studies reported on quality-of-life outcomes. Clinical experience suggests that adjuvant endocrine therapy is associated with a reasonably high quality of life for most patients, notwithstanding well-known treatment-related adverse effects including climacteric, musculoskeletal, and genitourinary symptoms. Clinicians familiar with the use of these agents are encouraged to gauge health-related quality of life among patients receiving adjuvant endocrine therapy to determine how treatment may be affecting them.

\section{Analysis by Menopausal Status}

Tamoxifen is active regardless of menopausal status and is the only recommended agent for women who are pre- or perimenopausal. ${ }^{5}$ Three studies presented analyses among subsets of patients defined by baseline menopausal status. ${ }^{9,14,15}$ These studies did not show any qualitative difference in outcome for longer duration tamoxifen treatment between women who were pre- or postmenopausal at baseline. In ATLAS, in particular, women who were pre- or perimenopausal derived substantial benefit from extended therapy with tamoxifen.

Clinical interpretation. Adjuvant endocrine therapy is a mainstay of therapy for women with ER-positive breast cancer. This guideline update reflects emerging data on the duration of tamoxifen treatment. There have been a total of five studies of tamoxifen treatment for longer than 5 years. The two largest studies with the longest reported follow-up now show a breast cancer survival advantage with longer durations of tamoxifen use; namely, 10 years of therapy. The beneficial effects of tamoxifen become more pronounced with longer follow-up, such that a minimum of 5 years of extended treatment (that is, 10 years since diagnosis) is needed to see clinical advantages. In addition to modest gains in survival, extended therapy with tamoxifen for a total of 10 years as compared with 5 years was associated with lower risk of breast cancer recurrence and a lower risk of contralateral breast cancer. Extended tamoxifen did not affect non-breast cancer mortality in the studies examined. Consistent with previous reports on the effects of adjuvant endocrine therapy, only patients with ER-positive tumors appear to benefit from extended therapy with tamoxifen.

Adverse effects reported by investigators of both 5- and 10-year duration tamoxifen, including menopausal symptoms and the rare risks of endometrial cancer and thromboembolism, appear to persist with longer durations of treatment. However, no new adverse effects specifically associated with longer durations of tamoxifen were identified in the current review. Clinical experience suggests that many patients have ongoing menopausal symptoms with extended duration of tamoxifen therapy, but the current evidence is not definitive.

Previous ASCO guidelines have recommended treatment of premenopausal women with 5 years of tamoxifen, and of postmenopausal women with a minimum of 5 years of adjuvant therapy using either an $\mathrm{AI}$ or a sequence of tamoxifen followed by an AI. ${ }^{5}$ As discussed in the 2010 guideline, AIs are an option only for women who are postmenopausal. On the basis of the emerging data outlined in this 
Focused Update, the Committee made separate recommendations for women who are pre- and postmenopausal.

Women who are postmenopausal who receive tamoxifen as initial treatment are candidates for extended adjuvant endocrine treatment either by continuing tamoxifen for a total of 10 years, or by switching to an AI. Qualitatively, there was no difference in the benefit (or lack thereof) seen with extended tamoxifen therapy in the subgroups defined by menopausal status. As there are no data for the efficacy or safety of AI therapy in excess of 5 years, 5 years remains the appropriate duration of AI treatment, whether begun as initial therapy or after prior tamoxifen.

Of the five trials analyzed in this guideline update, three $9,10,15$ showed positive results for extended tamoxifen treatment beyond 5 years, and two ${ }^{12-14}$ did not. It is not known what accounts for these discordant findings. The Update Committee noted that the trials with the largest sample size and longest follow-up ${ }^{9,10}$ yielded results with marked clinical benefit and, accordingly, made recommendations that predominantly reflect the scale and scope of those studies. An upcoming meta-analysis from the Early Breast Cancer Trialists Collaborative Group may provide additional insights.

For clinicians and patients, there are two distinct questions regarding optimal adjuvant endocrine therapy. One centers on the optimal initial treatment choice for women who are postmenopausal, who could receive either tamoxifen or an AI. The other centers on the optimal duration of adjuvant treatment. This guideline update has focused on the question of duration, specifically on extended endocrine therapy beyond 5 years of tamoxifen. On the basis of previous reports discussed in the 2010 guideline, extended adjuvant endocrine therapy with an $\mathrm{AI}$ after 5 years of tamoxifen reduces the risk of cancer recurrence in women who are postmenopausal, and the data reviewed in this update show that extended therapy with tamoxifen does the same. It is not known which strategy is preferred; tamoxifen and AIs have different adverse effect profiles that might reasonably inform that choice, and patient preferences based on the tolerability of these agents in individual women should be considered. Tamoxifen is effective regardless of menopausal status. Thus, whereas AI is an appropriate treatment option for women who are certainly menopausal, tamoxifen is the treatment of choice for pre- or perimenopausal women; women whose menopausal status is uncertain because of tamoxifen treatment, hysterectomy, or other medical circumstances; or women who do not tolerate AI treatment.

The Update Committee cautioned against longer durations of treatment when neither safety nor efficacy data were available. At present, such data are only available for up to 5 years of adjuvant endocrine therapy with AIs, and up to 10 years of adjuvant endocrine therapy with tamoxifen or with the cumulative sequence of tamoxifen and AIs. Ongoing clinical trials are comparing extended durations of AI therapy beyond 5 years to address questions of treatment duration.

There are no new data regarding the optimal initial endocrine treatment for women who are postmenopausal, whether that should be tamoxifen or an AI. The 2010 Update Committee recommendation of initial treatment with an AI or with tamoxifen to be followed by an AI remains valid. The Update Committee notes, however, that the accumulating data suggest that longer durations of endocrine therapy (either tamoxifen or tamoxifen followed by an AI) are associated with lower risk of cancer recurrence. On the basis of those trends, the Update Committee encourages patients and clinicians to embark on adjuvant endocrine treatment with an eye toward longer durations of therapy, not to exceed 10 years. Patients and their clinical teams should review adverse effect profiles and compliance with medications regularly, and work to find agents that patients find tolerable and will reliably take as medication.

The Update Committee acknowledges that the recommendations for adjuvant endocrine therapy continue to suggest different total durations of treatment based on the initial treatment strategy. At present, data are available regarding women who are postmenopausal or premenopausal who begin treatment with tamoxifen. However, for postmenopausal women who start treatment with an AI, there are no data showing that longer durations are clinically effective, nor are there efficacy data for switching to tamoxifen after 5 years of an AI. The Update Committee anticipates that data from ongoing trials will eventually clarify this discordance in total treatment duration.

Well-established clinical factors including tumor size; nodal status; ER, PgR, and HER2 biomarkers ${ }^{16}$; and molecular diagnostic assays ${ }^{17}$ serve as prognostic factors for breast cancer recurrence. However, there are no robust specific clinical or biomarker measures that selectively predict early versus late recurrence, nor predict whether tamoxifen or AI therapy would be appropriate treatment, nor determine whether longer durations of adjuvant endocrine therapy are clinically indicated. Identifying such markers is an important research priority. Extensive clinical data, specifically including data from trials of extended adjuvant treatment, indicate that smaller and/or node-negative cancers are at lower risk for recurrence after 5 years of adjuvant endocrine therapy than are larger and/or node-positive tumors. ${ }^{9,18-21}$ For this reason, the magnitude of benefit of extended durations of adjuvant therapy is certainly lower for patients with stage I cancers than for those with higher stage tumors. Although patients with favorable prognosis tumors may experience more risk than benefit with extended adjuvant treatment, the clinicopathological factors for defining such patients are not established. Some of the benefit of extended adjuvant treatment is derived from prevention of in-breast recurrences and/or second cancers. ${ }^{9,18-22}$ Women who have undergone bilateral mastectomy are not at risk for these occurrences, reducing their likely benefits from extended therapy. Considerations of benefit and risk on the basis of stage and the adverse effects experienced by a given patient may help clinical teams make individualized recommendations on the appropriateness of ongoing treatment for a specific woman.

Women receiving extended adjuvant endocrine therapy are at risk for ongoing adverse effects, such as menopausal symptoms, and less common, but more serious, adverse effects. Tamoxifen is associated with risks of thromboembolism and uterine cancer. AIs are associated with ongoing risk of osteoporosis. Clinicians should monitor patients for sequelae of treatment according to established guidelines. $^{23}$

Compliance to, and persistence with, adjuvant endocrine therapy for breast cancer is a clinical challenge. Patient adverse effects, personal health preferences, and out-of-pocket costs all contribute to nonadherence with adjuvant endocrine therapy. Given the importance of adjuvant endocrine treatment for improving overall survival in women with ER-positive breast cancer, clinicians are encouraged to inquire diligently about treatment compliance and treatment-related adverse effects and to pursue interventions known to mitigate adverse effects and enhance adherence. 
COMMENTARY ON ADHERENCE AND PERSISTENCE

The purpose of this section is to update the section of the 2010 guideline update that addresses aspects of patient-provider communication in selecting and using adjuvant endocrine therapy, as well as barriers to adherence to treatment regimens (taking medication as prescribed) and persistence with the medication schedule (taking medication for the full duration prescribed). Clinicians and patients should discuss the risks of recurrence, incorporating tumor size and nodal status. The discussion should include the risks and benefits of each treatment option on an individualized basis, recognizing the limitations of the data for specific options. Although not part of the systematic review, separate literature searches were conducted, and the Update Committee's expert opinion informed this section. The data discussed are, in part, from a systematic review published by Murphy et al, ${ }^{24}$ as well as articles that were not included in the Murphy systematic review, and references in those and other publications. ${ }^{24}$ Some of the publications combined participants who took tamoxifen and/or AIs. None reported data for patients who received adjuvant endocrine therapy beyond 5 years.

Rates of nonpersistence (early discontinuation of medication) in women who start adjuvant endocrine therapy range from $20 \%$ to $50 \%$, as previously discussed in the 2010 guideline. ${ }^{5}$ Rates of compliance (conformity to prescribed dosing) average $74 \%$ to $84 \%$, and may diminish over time. These rates of adherence are comparable to those seen for use of other medications used chronically, such as antihypertensives, ${ }^{25,26}$ cholesterol-lowering agents, ${ }^{27,28}$ or antiepileptics. ${ }^{29,30}$

Factors that decrease adherence with endocrine therapy include extremes of age, follow-up by general practitioners as opposed to oncologists, treatment-related symptoms, and out-of-pocket expenses. ${ }^{24}$ Adverse effects are particularly important in precipitating early discontinuation of therapy. Patients who experience treatmentrelated adverse effects are more likely to discontinue adjuvant endocrine therapy, particularly during the first years. Addressing patient beliefs about the benefits and risks of medications is warranted in patient-provider interactions about the use of adjuvant endocrine therapy. Helping patients understand the rationale for therapy, and the likely adverse effects, is likely to enhance treatment compliance and persistence.

Potentially modifiable factors that may enhance treatment compliance and/or persistence include follow-up with oncology team; inquiring about adverse effects, including menopausal, musculoskeletal, and genitourinary symptoms, headaches and sleep issues ${ }^{31}$; addressing inadequate social support, including for the treatment decision $^{24}$; providing information on adverse effects; and inquiring about both attitudes about medication in general $^{32}$ and endocrine therapy in particular, and about access to medications, including financial barriers. Clinicians imparting knowledge about recurrence may also increase persistence, ${ }^{24}$ as may the provision of follow-up care in the oncology setting. ${ }^{24}$

\section{HEALTH DISPARITIES}

Although ASCO clinical practice guidelines represent expert recommendations on the best practices in disease management to provide the highest level of cancer care, it is important to note that many patients have limited access to medical care. Racial and ethnic disparities in health care contribute significantly to this problem in the United States and around the world. Racial/ethnic minority patients with cancer suffer disproportionately from comorbidities, experience more substantial obstacles to receiving care, are more likely to be uninsured, and are at greater risk of receiving poor-quality care than others. ${ }^{33-36}$ Many other patients lack access to care because of their geography and distance from appropriate treatment facilities.

Representation of people of color and/or low socioeconomic status in clinical trials of adjuvant endocrine therapy is low. To date, no evidence indicates differences in therapeutic benefit between black and white women who receive adjuvant tamoxifen in the clinical trials setting. The evidence regarding disparities is mixed. In studies reviewed by Murphy et al, ${ }^{24}$ black race was negatively associated with adherence, but this association was not found in other studies. ${ }^{37}$ Some studies suggest that women who belong to nonwhite racial/ethnic groups and/or low socioeconomic groups are less likely to receive guideline-concordant endocrine therapy. ${ }^{38}$ For example, in one study, $19 \%$ of patients who should have received adjuvant endocrine therapy did not. ${ }^{39}$ In contrast, another study found the likelihood of initiating and persisting with therapy favored Latinas and African Americans, ${ }^{32}$ although other studies cited by these authors showed a lack of differences. However, awareness of disparities in quality of care should be considered in the context of this clinical practice guideline, and health care providers should strive to deliver the highest level of cancer care to these vulnerable populations.

\section{MULTIPLE CHRONIC CONDITIONS}

Creating evidence-based recommendations to inform treatment of patients with additional chronic conditions, a situation in which the patient may have two or more such conditions-referred to as multiple chronic conditions (MCC) - is challenging. Patients with MCC are a complex and heterogeneous population, making it difficult to account for all of the possible permutations to develop specific recommendations for care. In addition, the best available evidence for treating index conditions, such as cancer, is often from clinical trials whose study selection criteria may exclude these patients in order to avoid potential interaction effects or confounding of results associated with MCC. As a result, the reliability of outcome data from these studies may be limited, thereby creating constraints for expert groups to make recommendations for care in this heterogeneous patient population. ${ }^{40}$

Because many patients for whom guideline recommendations apply present with MCC, any treatment plan needs to take into account the complexity and uncertainty created by the presence of MCC; this highlights the importance of shared decision making around guideline use and implementation. Therefore, in consideration of recommended care for the target index condition, clinicians should review all other chronic conditions present in the patient and take those conditions into account when formulating the treatment and follow-up plan (common chronic conditions for patients with breast cancer are listed in Data Supplement 5).

Taking the above considerations into account, practice guidelines should provide information on how to apply the recommendations for patients with MCC, perhaps as a qualifying statement for 
recommended care. This may mean that some or all of the recommended care options are modified or not applied, as determined by best practice in consideration of any MCC.

\section{GUIDELINE IMPLEMENTATION}

ASCO guidelines are developed for implementation across health settings. Barriers to implementation include the need to increase awareness of the guideline recommendations among front-line practitioners and cancer survivors, and also to provide adequate services in the face of limited resources. The guideline Bottom Line was designed to facilitate implementation of recommendations. This guideline will be distributed widely through the ASCO Practice Guideline Implementation Network. ASCO guidelines are posted on the ASCO Web site and most often published in Journal of Clinical Oncology and Journal of Oncology Practice.

\section{LIMITATIONS OF THE RESEARCH}

Limitations of the studies discussed in this guideline include differing amounts of median follow-up and the fact that the studies were performed in different eras, in part resulting in insufficient information to appraise the quality of three of the studies. ${ }^{10,13-15}$ Some of the populations in these studies did not have hormone receptor-positive breast cancer and/or their hormone receptor status was unknown. In addition, there are relatively few new data on adverse events for those who have received adjuvant tamoxifen for more than 5 years. The studies in this guideline provided insufficient data on adverse effects, especially climacteric and/or sexual adverse effects. Evidence on a broader set of adverse events could affect women's risk-benefit perceptions and willingness to take tamoxifen for more than 5 years, perhaps modulated by age and menopausal status. The studies did not report on and/or did not measure health-related quality of life; at the time of the development of this guideline, results by menopausal status were not available, and there are few data on extended durations of AIs.

\section{FUTURE DIRECTIONS}

The Update Committee recommends research in participants receiving more than a five-year duration of AIs and on the issue of duration for those who took tamoxifen for less than 5 years. In addition, more information is needed regarding predictors of risk of recurrence and of likely treatment benefit based on tumor stage, biomarkers, and time since diagnosis. It is important to identify strategies that are likely to promote the use of effective endocrine treatments among women, especially given the recommendations for longer durations of therapy.

\section{ADDITIONAL RESOURCES}

More information, including Data Supplement with additional evidence tables, a Methodology Supplement with information about evidence quality and strength of recommendations, slide sets, and clinical tools and resources, is available at www.asco.org/ guidelines/endocrinebreast. Patient information is available there and at www.cancer.net.

\section{AUTHORS' DISCLOSURES OF POTENTIAL CONFLICTS OF INTEREST}

Although all authors completed the disclosure declaration, the following author(s) and/or an author's immediate family member(s) indicated a financial or other interest that is relevant to the subject matter under consideration in this article. Certain relationships marked with a " $U$ " are those for which no compensation was received; those relationships marked with a "C" were compensated. For a detailed description of the disclosure categories, or for more information about ASCO's conflict of interest policy, please refer to the Author Disclosure Declaration and the Disclosures of Potential Conflicts of Interest section in Information for Contributors. Employment or Leadership Position: None Consultant or Advisory Role: Eric P. Winer, AstraZeneca (U), Novartis (U) Stock Ownership: None Honoraria: Karen Gelmon, AstraZeneca, Novartis Research Funding: Vered Stearns, Abbvie, Celgene, Medimmune, Merck, Novartis, Pfizer Expert Testimony: None Patents, Royalties, and Licenses: None Other Remuneration: None

\section{AUTHOR CONTRIBUTIONS}

\author{
Administrative support: Sarah Temin \\ Manuscript writing: All authors \\ Final approval of manuscript: All authors
}

\section{REFERENCES}

1. College of American Pathologists: Breast Cancer: Invasive Ductal Carcinoma. 2011http://www.cap.org/ apps/docs/reference/myBiopsy/BreastInvasiveDuctal Carcinoma.pdf

2. WebMD: Types of Breast Cancer: ER Positive, HER2 Positive, and Triple Negative. http:// www.webmd.com/breast-cancer/breast-cancer-typeser-positive-her2-positive, 2012

3. National Cancer Institute: Hormone Therapy for Breast Cancer. 2012http://www.cancer.gov/ cancertopics/factsheet/Therapy/hormone-therapy-breast

4. Caring 4 Cancer. Estrogen Receptor Status May Determine Chemotherapy Use. 2010 https:// www.caring4cancer.com/go/breast/treatments/ chemotherapy/estrogen-receptor-status-may-determinechemotherapy-use.htm
5. Burstein HJ, Prestrud AA, Seidenfeld J, et al: American Society of Clinical Oncology clinical practice guideline: Update on adjuvant endocrine therapy for women with hormone receptorpositive breast cancer. J Clin Oncol 28:3784-3796, 2010

6. Winer EP, Hudis $\mathrm{C}$, Burstein $\mathrm{HJ}$, et al: American Society of Clinical Oncology technology assessment working group update: Use of aromatase inhibitors in the adjuvant setting. J Clin Oncol 21: 2597-2599, 2003

7. Winer EP, Hudis $C$, Burstein $\mathrm{HJ}$, et al: American Society of Clinical Oncology technology assessment on the use of aromatase inhibitors as adjuvant therapy for women with hormone receptor-positive breast cancer: Status report 2002. J Clin Oncol 20:3317-3327, 2002

8. Winer EP, Hudis $C$, Burstein $\mathrm{HJ}$, et al: American Society of Clinical Oncology technology assess- ment on the use of aromatase inhibitors as adjuvant therapy for postmenopausal women with hormone receptor-positive breast cancer: Status report 2004. $\mathrm{J}$ Clin Oncol 23:619-629, 2005

9. Davies C, Pan H, Godwin J, et al: Long-term effects of continuing adjuvant tamoxifen to 10 years versus stopping at 5 years after diagnosis of oestrogen receptor-positive breast cancer: ATLAS, a randomised trial. Lancet 381:805-816, 2013

10. Gray RG, Rea D, Handley K, et al: ATTom: Long-term effects of continuing adjuvant tamoxifen to 10 years versus stopping at 5 years in 6,953 women with early breast cancer. J Clin Oncol 31, 2013 (suppl; abstr 5)

11. Shiffman RN, Michel G, Rosenfeld RM, et al: Building better guidelines with BRIDGE-Wiz: Development and evaluation of a software assistant to promote clarity, transparency, and implementability. J Am Med Inform Assoc 19:94-101, 2012 
12. Fisher $B$, Dignam J, Bryant $J$, et al: Five versus more than 5 years of tamoxifen for lymph nodenegative breast cancer: Updated findings from the National Surgical Adjuvant Breast and Bowel Project B-14 randomized trial. J Natl Cancer Inst 93:684690, 2001

13. Stewart $H J$, Forrest $A P$, Everington $D$, et al: Randomised comparison of 5 years of adjuvant tamoxifen with continuous therapy for operable breast cancer. The Scottish Cancer Trials Breast Group. Br J Cancer 74:297-299, 1996

14. Stewart HJ, Prescott RJ, Forrest AP: Scottish adjuvant tamoxifen trial: A randomized study updated to 15 years. J Natl Cancer Inst 93:456-462, 2001

15. Tormey DC, Gray R, Falkson HC: Postchemotherapy adjuvant tamoxifen therapy beyond 5 years in patients with lymph node-positive breast cancer. Eastern Cooperative Oncology Group. J Natl Cancer Inst 88:1828-1833, 1996

16. Hammond ME, Hayes DF, Wolff $A C$, et al: American Society of Clinical Oncology/College of American Pathologists guideline recommendations for immunohistochemical testing of estrogen and progesterone receptors in breast cancer. J Oncol Pract 6:195-197, 2010

17. Harris L, Fritsche $H$, Mennel $R$, et al: American Society of Clinical Oncology 2007 update of recommendations for the use of tumor markers in breast cancer. J Clin Oncol 25:5287-5312, 2007

18. Goss PE: Letrozole in the extended adjuvant setting. Breast Cancer Res Treat 105:45-53, 2007 (suppl 1)

19. Goss PE, Ingle JN, Martino S, et al: Efficacy of letrozole extended adjuvant therapy according to estrogen receptor and progesterone receptor status of the primary tumor: National Cancer Institute of Canada Clinical Trials Group. J Clin Oncol 25:20062011, 2007

20. Goss PE, Ingle JN, Martino S, et al: A randomized trial of letrozole in postmenopausal women after 5 years of tamoxifen therapy for early-stage breast cancer. N Engl J Med 349:1793-1802, 2003
21. Ingle JN, Tu D, Pater JL, et al: Intent-to-treat analysis of the placebo-controlled trial of letrozole for extended adjuvant therapy in early breast cancer: NCIC CTG. Ann Oncol 19:877-882, 2008

22. Mamounas EP, Jeong JH, Wickerham $\mathrm{DL}$, et al: Benefit from exemestane as extended adjuvant therapy after 5 years of adjuvant tamoxifen: Intention-to-treat analysis of the National Surgical Adjuvant Breast And Bowel Project B-33 trial. J Clin Oncol 26:1965-1971, 2008

23. Khatcheressian JL, Hurley $P$, Bantug $E$, et al: Breast cancer follow-up and management after primary treatment: American Society of Clinical Oncology clinical practice guideline update. J Clin Oncol 31:961-965, 2013

24. Murphy CC, Bartholomew LK, Carpentier MY, et al: Adherence to adjuvant hormonal therapy among breast cancer survivors in clinical practice: A systematic review. Breast Cancer Res Treat 134: 459-478, 2012

25. Muszbek N, Brixner D, Benedict A, et al: The economic consequences of noncompliance in cardiovascular disease and related conditions: A literature review. Int J Clin Pract 62:338-351, 2008

26. Naderi SH, Bestwick JP, Wald DS: Adherence to drugs that prevent cardiovascular disease: Metaanalysis on 376,162 patients. Am J Med 125:882887; e1, 2012

27. Gomez Sandoval YH, Braganza MV, Daskalopoulou SS: Statin discontinuation in high-risk patients: A systematic review of the evidence. Curr Pharm Des 17:3669-3689, 2011

28. Perreault S, Ellia L, Dragomir A, et al: Effect of statin adherence on cerebrovascular disease in primary prevention. Am J Med 122:647-655, 2009

29. Sherrill B, Halpern M, Khan $S$, et al: Single-pill vs free-equivalent combination therapies for hypertension: A meta-analysis of health care costs and adherence. J Clin Hypertens (Greenwich) 13:898909, 2011

30. Tellez-Zenteno JF, Hernandez-Ronquillo L, Moien-Afshari F: Discontinuation of antiepileptic drugs after successful surgery: Who and when? Epileptic Disord 14:363-370, 2012

31. Aiello Bowles EJ, Boudreau DM, Chubak J, et al: Patient-reported discontinuation of endocrine therapy and related adverse effects among women with early-stage breast cancer. J Oncol Pract 8:e149e157, 2012

32. Friese CR, Pini TM, Li $Y$, et al: Adjuvant endocrine therapy initiation and persistence in a diverse sample of patients with breast cancer. Breast Cancer Res Treat 138:931-939, 2013

33. American Cancer Society: Cancer Facts \& Figures for African Americans 2011-2012. Atlanta, GA, American Cancer Society, 2011

34. Centers for Disease Control and Prevention, National Program of Cancer Registries: United States Cancer Statistics: 1999-2008 Incidence and Mortality Data. 2012 www.cdc.gov/uscs

35. Howlader N, Noone AM, Krapcho M, et al: SEER Cancer Statistics Review, 1975-2009 (Vintage 2009 Populations). http://seer.cancer.gov/csr/1975_2009_pops09/

36. Mead H, Cartwright-Smith L, Jones $\mathrm{K}$, et al: Racial and Ethnic Disparities in U.S. Health Care: A Chartbook. New York, NY, The Commonwealth Fund 2008

37. Yen TW, Czypinski LK, Sparapani RA, et al: Socioeconomic factors associated with adjuvant hormone therapy use in older breast cancer survivors. Cancer 117:398-405, 2011

38. Wu XC, Lund MJ, Kimmick GG, et al: Influence of race, insurance, socioeconomic status, and hospital type on receipt of guideline-concordant adjuvant systemic therapy for locoregional breast cancers. J Clin Oncol 30:142-150, 2012

39. Griggs JJ: Role of nonclinical factors in the receipt of high-quality systemic adjuvant breast cancer treatment. J Clin Oncol 30:121-124, 2012

40. American Geriatrics Society Expert Panel on the Care of Older Adults with Multimorbidity: Patient-centered care for older adults with multiple chronic conditions: A stepwise approach from the American Geriatrics Society. J Am Geriatr Soc, 2012 


\section{Acknowledgment}

The Update Committee wishes to thank Christopher G. Azzoli, MD, Bryan J. Schneider, MD, and the full Clinical Practice Guidelines Committee for their thoughtful reviews and insightful comments on this guideline document; Anne A. Prestrud for assistance with the systemic review; and Christina Lacchetti for further assistance.

\section{Appendix}

Table A1. Adjuvant Endocrine Therapy for Women With Hormone Receptor-Positive Breast Cancer Update Committee Membership

\begin{tabular}{|c|c|c|}
\hline Name (and designation) & Affiliation/Institution & Role/Area of Expertise \\
\hline Harold J. Burstein, MD, PhD & Dana-Farber Cancer Institute, Boston, MA & Medical oncology \\
\hline Jennifer J. Griggs, MD MPH & University of Michigan, Ann Arbor, MI & Medical oncology \\
\hline Holly Anderson & Breast Cancer Coalition of Rochester, Rochester, NY & Patient representative \\
\hline Thomas A. Buchholz, MD & MD Anderson Cancer Center, Houston, TX & Radiation oncology \\
\hline Nancy E. Davidson, MD & University of Pittsburgh Cancer Institute and UPMC CancerCenter, Pittsburgh, PA & Medical oncology \\
\hline Karen E. Gelmon, MD & British Columbia Cancer Agency, Vancouver, BC & Medical oncology \\
\hline Sharon H. Giordano, MD & MD Anderson Cancer Center, Houston, TX & Medical oncology \\
\hline Clifford A. Hudis, MD & Memorial Sloan Kettering Cancer Center, New York, NY & Medical oncology \\
\hline Diana Rowden, MA & Dallas, TX & Patient advocate \\
\hline Alexander J. Solky, MD & Interlakes Onc and Hem PC, Rochester, NY & Community oncology \\
\hline Vered Stearns, MD & Johns Hopkins School of Medicine, Baltimore, MD & Medical oncology \\
\hline Eric P. Winer, MD & Dana-Farber Cancer Institute, Boston, MA & Medical oncology \\
\hline
\end{tabular}

NOTE. ASCO Staff: Sarah Temin, MSPH. 